\title{
MODELO DE LOCALIZACIÓN ÓPTIMA DE ACTIVIDADES NO DESEADAS APLICADO A LOS RESIDUOS SÓLIDOS EN LA REGIÓN METROPOLITANA
}

\section{AN OPTIMAL LOCATION MODEL OF UNDESIRABLE FACILITIES APPLIED TO THE SOLID WASTES IN THE METROPOLITAN REGION}

\author{
Marcos Medina Tapia ${ }^{1} \quad$ Jorge Cerda Troncoso ${ }^{1}$ \\ Recibido el 21 de junio de 2006, aceptado el 6 de noviembre de 2007 \\ Received: June 21, 2006 Accepted: November 6, 2007
}

\begin{abstract}
RESUMEN
El trabajo analiza los principios y estructuras de modelación de localización óptima de actividades indeseables (obnoxious location models), revisando criterios de "eficiencia espacial" en la localización, y "justicia espacial" en la distribución de externalidades. La eficiencia se logra con modelos de localización-asignación, optimizando el costo total (plant location). Para la justicia se calibran curvas de rechazo con métodos econométricos. Finalmente todo se optimiza en una estructura multiobjetivo ponderada. Este modelo mixto se aplica a la localización de estaciones de transferencia y rellenos sanitarios en la Región Metropolitana, obteniendo localizaciones óptimas (económica y socialmente), y asignaciones óptimas de residuos sólidos comunales.
\end{abstract}

Palabras clave: Localización óptima, actividades no deseadas, rellenos sanitarios.

\begin{abstract}
This work analyses the principles and structures of optimal location models of obnoxious activities, by studying criterion of spatial efficiency in the location, and spatial justice in the distribution of externalities. The efficiency is obtained through the Plant Location model (a location-allocation model). For the justice, opposition curves are fitted using econometrics models. Finally, everything is optimised in a weighted multi-objective structure. This mixed model is applied to the location of transfer stations and landfill operations in the Metropolitan Area in Santiago-Chile. The results give optimal locations (economic and social), and optimal allocation of municipal solid waste.
\end{abstract}

Keywords: Optimal location, undesirable facilities, Landfill.

\section{INTRODUCCIÓN}

Los modelos de localización de actividades indeseables (obnoxious location models) se caracterizan por tener dos objetivos que optimizar. El primero requiere que la actividad se localice lo más lejos posible para evitar las externalidades negativas en la población. El segundo requiere que la actividad se localice lo más cerca posible para lograr algún tipo de eficiencia operativa. Aplicaciones típicas de este tipo de problemas son la localización de centrales nucleares, plantas de tratamiento de agua servidas, etc. [6].

Las externalidades negativas de una actividad socialmente indeseable producen un efecto de rechazo que ha sido denominado, NIMBY ("Not in My Backyard"), LULU ("Locally Unwanted Land Use"), e incluso la negativa absoluta a la localización como BANANA ("Build Absolutely Nothing at All Near Anybody"), entre otras [10].

Según la literatura, los modelos de localización para actividades indeseables consideran dos principios: el primero, es el de eficiencia espacial en la localización y, por otro lado, el de justicia espacial debido a la distribución de externalidades [1].

El principio de eficiencia espacial garantiza que los costos totales de localización de la actividad sean mínimos. Los costos totales consideran la instalación y el transporte (en función del volumen transportado) [1].

\footnotetext{
1 Departamento de Ingeniería Geográfica. Universidad de Santiago de Chile. Av. Ecuador 3519. Casilla 10233. Santiago, Chile. E-mail: marcosmedina@usach.cl, jcerdat@gmail.com
} 
Por lo tanto, la eficiencia del sistema busca el equilibrio, minimizando la distancia entre los generadores y los equipamientos y, al mismo tiempo, la maximización de la distancia entre la población y las instalaciones.

El principio de justicia espacial se refiere al grado de igualdad en la distribución de los servicios de cada equipamiento hacia la población. En la localización de actividades indeseables, la justicia espacial es representada por el grado en que una población comparte los riesgos o molestias que dichas instalaciones representan. Para ello es necesario minimizar estas externalidades, por lo tanto, la localización más justa es aquella en que existan las menores diferencias en la forma en que los diversos sectores de la población se ven afectados por el emplazamiento de una instalación y, por ende, el menor costo para el Sistema [1].

A pesar de su indiscutible relevancia en el proceso de localización de instalaciones indeseables, la justicia espacial ha sido y es un concepto difícil de medir y más complicado de aplicar.

La temática de Residuos Sólidos Domiciliarios, en el último tiempo, ha tenido un carácter predominante en el país, particularmente en la Región Metropolitana de Santiago. Pero el tema de Residuos Sólidos es más que el problema en "dónde botar la basura", porque es posible distinguir diferentes partes o etapas dentro del proceso, como son la Generación, Recolección, Transporte, Tratamiento, Recuperación y Reducción de volumen, y Disposición Final, las cuales interrelacionan en forma secuencial, teniendo el objetivo de hacerse cargo de los Residuos Sólidos generados por un centro poblado, ya sea tratándolos, reduciéndolos o disponiéndolos en forma terminal [14].

El problema de la Disposición Final de Residuos Sólidos Domiciliarios (RSD) se enmarca, como se mencionó, dentro del Sistema, en el que intervienen diferentes elementos espaciales, como son: los Centros de Población (Comunas, Ciudades o Pueblos, en fin, regiones generadoras de RSD), y Sitios de Disposición Final, como son los Rellenos Sanitarios. A esto es posible agregar las Estaciones de Transferencia que permiten una minimización de costos de transporte.

Cada uno de los elementos mencionados, ya sea Centros de Población, Estaciones de Transferencia y Rellenos Sanitarios presentan una localización o una potencial localización y, por ende, una distribución en el espacio. Cada uno de los elementos se encuentra interrelacionado con otros elementos, es decir, un
Relleno Sanitario debe considerar la localización de las Estaciones de Transferencia y, al mismo tiempo, las Estaciones de Transferencia deben considerar la localización que tienen los Centros de Población, como también los Rellenos deben considerar la localización de la Población. Las vías que unen los Centros de Población con las Estaciones de Transferencia y éstas con los Rellenos Sanitarios generan una red que permite el transporte de los RSD.

Finalmente, el objetivo general de este trabajo fue construir un modelo de optimización multiobjetivo, considerando optimización en costos y en distribución de externalidades espaciales, de manera de optimizar la localización de estaciones de transferencia y Rellenos Sanitarios en la Región Metropolitana de Santiago.

\section{MODELO APLICADO}

Para la implementación del modelo se utilizó la Red Vial que corresponde a la unión de dos redes, la urbana (ESTRAUS) y la interurbana (de vías relevantes según clasificación MOP).

Las zonas generadoras de RSD fueron las 52 comunas de la Región Metropolitana, concebidas como un centroide que concentra todas las características comunales y que se conecta a la red vial en el nodo más cercano a éste.

Los Sitios Candidatos fueron los sitios aptos propuestos por el Gobierno Regional, en base a un estudio que considera factores netamente ambientales. Así se consideró como candidatos a un total de 6 estaciones de transferencia y 8 rellenos sanitarios $[4,8]$.

Como equipamientos existentes se consideraron los Rellenos Sanitarios de Loma Los Colorados (Til Til), Santa Marta (Talagante) y Santiago Poniente (Maipú). Las Estaciones de Transferencia que se consideraron en operación son: Estación Quilicura (Quilicura) y Puerta Sur (San Bernardo).

Para la predicción de los volúmenes comunales de RSD se aplicó la metodología de Box-Jenkins, el cual permite determinar los parámetros de modelos de tipo estocástico que permiten analizar series temporales para realizar predicciones mediante procesos autorregresivos y medias móviles, tipo AR(p), MA(q), ARMA(p,q) y ARIMA, a través de cuatro etapas: identificación, estimación, verificación de diagnóstico y predicción [12]. Para estimar el error, se hicieron predicciones a las series temporales del total 
regional de RSD y, en forma independiente, a las series de residuos sólidos comunales.

Para la estimación de los costos de instalación de las alternativas de Rellenos Sanitarios se utilizará la metodología de la CEPIS (Centro Panamericano de Ingeniería Sanitaria y Ciencias del Ambiente, OPS), que considera costos de adquisición del predio, costos de construcción, costos de operación y costos de clausura (ya que los rellenos se caracterizan por tener costos a largo plazo, posterior a su cierre). Para los costos de una Estación de Transferencia se utilizaron metodologías bibliográficas ad hoc, que considera tanto costos de instalación como de operación, no considerándose en este caso costos por clausura [9].

Los costos de transporte se tomaron de estudios específicos, diferenciándose los costos para camiones recolectores, y para camiones tipo silos (los que transportan los residuos compactados entre las estaciones de transferencia y los rellenos sanitarios). Los costos de transporte se expresaron en función de la distancia, con lo cual se pobló la red vial de modelación. Sobre esta red se calcularon rutas mínimas múltiples (con algoritmo de Floyd), entre todas las zonas generadoras y los rellenos sanitarios candidatos, zonas generadoras y estaciones de transferencia candidatas, y finalmente entre estaciones de transferencia y rellenos sanitarios.

\section{Eficiencia económica (modelo de costos)}

La modelación de costos se hizo a través de la estructura del modelo de localización óptima de costo fijo, o también conocido como Plant Location, donde se incluye una componente de costo de instalación de equipamiento (relleno sanitario y estación de transferencia) diferenciado entre el tamaño del equipamiento. Por otro lado, la formulación original de este modelo incluye la variable de asignación, la cual corresponde al flujo o volumen de RSD asignado y optimizado en función del costo de transporte de RSD para cada par origen-destino.

\section{Eficiencia social (externalidades en avalúos)}

Se denominó como población que percibe Externalidades de un equipamiento (Relleno Sanitario y/o Estación de Transferencia), a la que se encuentra dentro del área de influencia delimitada por el "Modelo de Percepción de Externalidades" construido para este estudio.

El efecto de la externalidad se obtuvo de la percepción de pérdida de valor de la vivienda, por parte de los habitantes del entorno del emplazamiento.

Para lo anterior, se aplicó una encuesta a la población, por muestreo estratificado, y con significancia espacial de la muestra. En dicha encuesta se consultaron variables de control y de percepción de externalidades económicas, todo en función de la distancia a los rellenos y estaciones actualmente existentes. Con la información obtenida se calibró un modelo econométrico (uno para rellenos y otro para estaciones) que explica la percepción de "devalúo de la propiedad" en función de la distancia.

Con el modelo calibrado se calculó el costo social, producto del devalúo de las viviendas que se produce para cada uno de los emplazamientos candidatos.

\section{Modelo de localización óptima multiobjetivo}

Las decisiones de optimización tienen que ver con variables de localización de y asignación de volúmenes de RSD.

La formulación multiobjetivo combina los costos económicos (localización y asignación de RSD), con los costos sociales (devalúos totales de viviendas), a través de ponderadores.

Los parámetros del modelo tienen que ver con volúmenes generados, costos de instalación, costos unitarios de transporte, capacidades y costos de devaluación de viviendas.

La formulación final se presenta a continuación:

\begin{tabular}{|ll|}
\hline $\mathrm{z}_{\mathrm{m}}^{\mathrm{n}}$ & $=1$, si un equipamiento de ET de tamaño (alternativa) $\mathrm{n}$ es localizado en el sitio m, 0 en otro caso. \\
$\mathrm{y}_{\mathrm{j}}^{\mathrm{k}}$ & $=1$, si un equipamiento de RS de tamaño (alternativa) $\mathrm{k}$ es localizado en el sitio j, 0 en otro caso. \\
\hline $\mathrm{t}_{\mathrm{ij}}$ & $=$ residuos destinados desde el centro de población $\mathrm{i}$ al RS $\mathrm{j}$. \\
$\mathrm{u}_{\mathrm{im}}$ & $=$ residuos destinados desde el centro de población i a la ET m. \\
$\mathrm{x}_{\mathrm{mj}}$ & $=$ residuos destinados desde la ET $\mathrm{m}$ al RS $\mathrm{j}$. \\
\hline
\end{tabular}

Figura 1. Variables de decisión del modelo. 


\begin{tabular}{|l|l|}
\hline $\mathrm{D}_{\mathrm{i}}$ & $=$ residuos generados (demanda) por el centro de población $i$. \\
\hline $\mathrm{a}_{\mathrm{k}}$ & $\begin{array}{l}\text { = capacidad de un equipamiento de RS de tamaño } k . \\
\text { = capacidad de un equipamiento de ET de tamaño } n .\end{array}$ \\
$\mathrm{b}_{\mathrm{n}}$ & $=$ costo total de un equipamiento de ET de tamaño $n$ en el sitio $m$. \\
$\mathrm{C}(\mathrm{ET})_{\mathrm{m}}^{\mathrm{n}}$ & $=$ costo total de un equipamiento de RS de tamaño $k$ en el sitio $j$. \\
$\mathrm{C}(\mathrm{RS})_{\mathrm{j}}^{\mathrm{k}}$ & $=$ costo unitario de transporte desde el centro de población $i$ al sitio RS $j$. \\
\hline $\mathrm{CT}_{\mathrm{ij}}$ & $\begin{array}{l}\text { = costo unitario de transporte desde el centro de población } i \text { al sitio } m . \\
\mathrm{CT}_{\mathrm{im}}\end{array}$ \\
$\mathrm{CT}_{\mathrm{mj}}$ & $=$ costo unitario de transporte desde la ET m al RS $j$. \\
\hline $\mathrm{P} 1, \mathrm{P} 2$ & $=$ ponderaciones. \\
\hline
\end{tabular}

Figura 2. Parámetros requeridos por el Modelo.

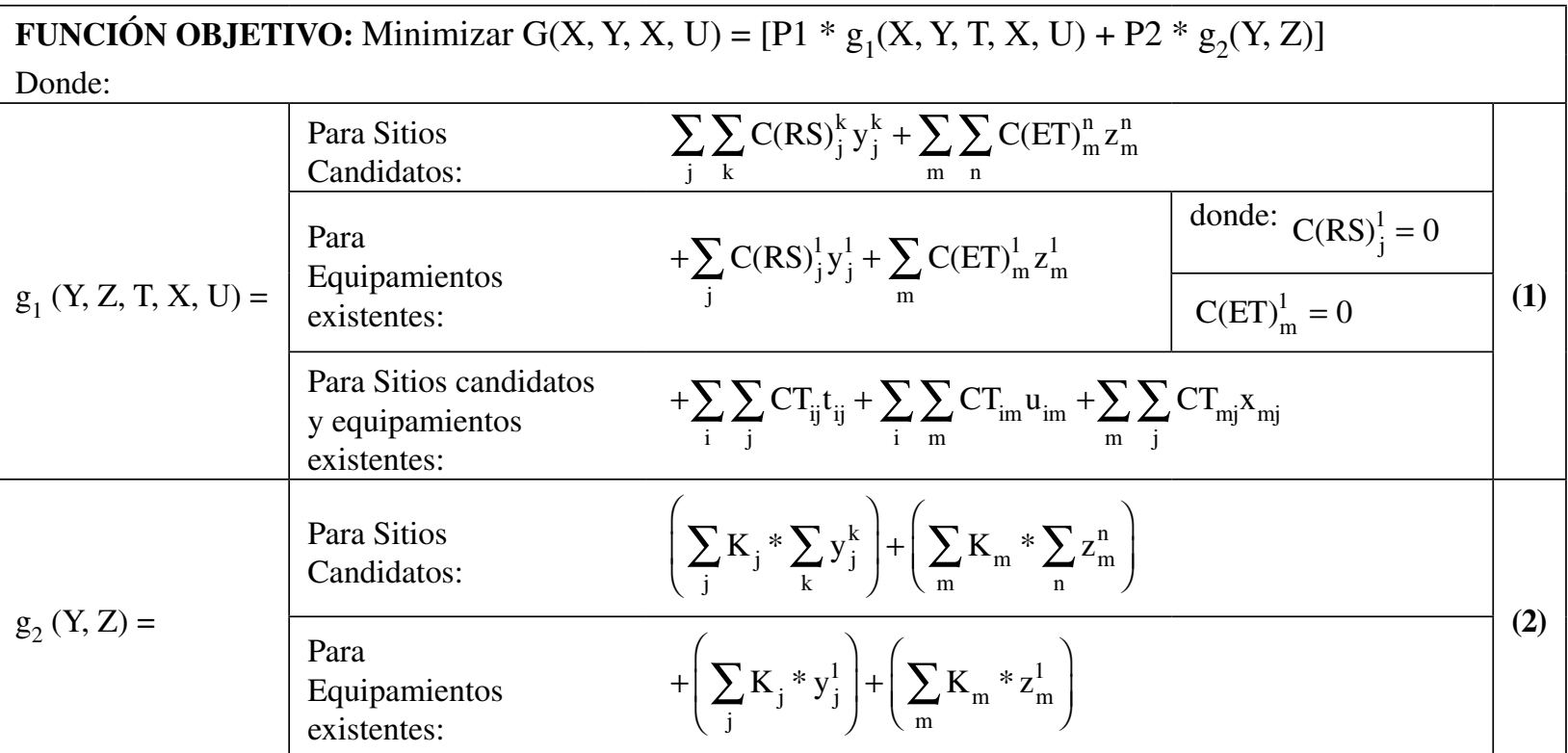

\begin{tabular}{|ccc|}
\hline Sujeto a: & $\forall \mathrm{m}$ & (3) \\
$\sum_{\mathrm{i}} \mathrm{u}_{\mathrm{im}}=\sum_{\mathrm{j}} \mathrm{x}_{\mathrm{mj}}$ & $\forall \mathrm{i}$ & (4) \\
$\sum_{\mathrm{j}} \mathrm{t}_{\mathrm{ij}}+\sum_{\mathrm{m}} \mathrm{u}_{\mathrm{im}}=\mathrm{D}_{\mathrm{i}}$ & $\forall \mathrm{j}$ & (5) \\
$\sum_{\mathrm{i}} \mathrm{t}_{\mathrm{ij}}+\sum_{\mathrm{m}} \mathrm{x}_{\mathrm{mj}} \leq \sum_{\mathrm{k}} \mathrm{a}_{\mathrm{k}} \mathrm{y}_{\mathrm{j}}^{\mathrm{k}}$ & $\forall \mathrm{m}$ & (6) \\
$\sum_{\mathrm{i}} \mathrm{u}_{\mathrm{im}} \leq \sum_{\mathrm{n}} \mathrm{b}_{\mathrm{n}} \mathrm{z}_{\mathrm{m}}^{\mathrm{n}}$ & $\forall \mathrm{m}$ & (7) \\
$\sum_{\mathrm{n}} \mathrm{z}_{\mathrm{m}}^{\mathrm{n}} \leq 1$ & $\forall \mathrm{j}$ & (8) \\
$\sum_{\mathrm{k}} \mathrm{y}_{\mathrm{j}}^{\mathrm{k}} \leq 1$ & & (9) \\
$\sum_{\mathrm{m}} \sum_{\mathrm{n}} \mathrm{z}_{\mathrm{m}}^{\mathrm{n}} \geq 0$ & &
\end{tabular}

Figura 3. Modelo de localización óptima de actividades no-deseadas (continúa). 


\begin{tabular}{|lccc|}
\hline \multicolumn{1}{|c|}{$\sum_{\mathrm{j}} \sum_{\mathrm{k}} \mathrm{y}_{\mathrm{j}}^{\mathrm{k}} \geq 0$} & & (10) \\
$\mathrm{t}_{\mathrm{ij}} \geq 0$ & $\forall \mathrm{i}, \mathrm{j}$ & (11) \\
\hline $\mathrm{u}_{\mathrm{im}} \geq 0$ & $\forall \mathrm{i}, \mathrm{m}$ & (12) \\
$\mathrm{x}_{\mathrm{mj}} \geq 0$ & $\forall \mathrm{m}, \mathrm{j}$ & (13) \\
& $\mathrm{y}_{\mathrm{j}}^{\mathrm{k}}=\mathrm{o}_{1}$ & $\forall \mathrm{j}, \mathrm{k}$ & (14) \\
& $\mathrm{z}_{\mathrm{m}}^{\mathrm{n}}=\mathrm{o}_{1}$ & $\forall \mathrm{m}, \mathrm{n}$ & (15) \\
\hline & $\sum_{\mathrm{i}} \mathrm{t}_{\mathrm{ij}}+\sum_{\mathrm{m}} \mathrm{x}_{\mathrm{mj}} \geq \sum_{\mathrm{k}} \mathrm{a}_{\mathrm{k}} \mathrm{y}_{\mathrm{j}}^{1}$ & $\forall \mathrm{j}$ & (16) \\
Para & $\sum_{\mathrm{i}} \mathrm{u}_{\mathrm{im}} \geq \sum_{\mathrm{n}} \mathrm{b}_{\mathrm{n}} \mathrm{z}_{\mathrm{m}}^{1}$ & $\forall \mathrm{m}$ & (17) \\
Equipamientos & $\mathrm{z}_{\mathrm{m}}^{1}=1$ & $\forall \mathrm{m}$ & (18) \\
existentes: & $\mathrm{y}_{\mathrm{j}}^{1}=1$ & $\forall \mathrm{j}$ & (19) \\
\hline
\end{tabular}

Figura 3. Modelo de localización óptima de actividades no-deseadas.

La función $\mathrm{g}_{1}$ corresponde a los costos económicos y la $\mathrm{g}_{2}$ a los costos sociales. Los ponderadores P1 y P2, que hacen competir los dos objetivos (económico y social) surgen, en primera instancia, de la aplicación del método delphi a un panel de expertos. Posteriormente se realizó un análisis de sensibilidad sobre estos parámetros.

Las restricciones dan cuenta de criterios de satisfacción de demandas (que se recogiera todo el RSD generados en las comunas), de capacidades respecto al transporte en camiones y a los rellenos y estaciones de balances (lo que entra a una ET debe ser igual a lo que sale), y de existencias (una ET o RS no puede servir si no existe).

La estrategia de modelación fue considerar dos cortes temporales (8 y 15 años) con estimación de RSD y dos escenarios de operación. El primer escenario de operación se denominó como "restrictivo", en el sentido que se obligó a que toda la asignación de RSD pase por una estación de transferencia. El segundo escenario, denominado "libre", deja libre la elección de pasar o no por una ET.

Para resolver este problema se implementó una aplicación en plataforma SIG (Sistema de Información Geográfica), con una interfase al solver LINDO v5.3. Esto permite tener una cartografía digital en donde se pueden elegir los sitios candidatos a considerar, para finalmente visualizar espacialmente el resultado de la optimización con LINDO. El método de optimización fue Branch and Bounds.

\section{Resultados obtenidos}

Para las externalidades sociales (devalúo) se utilizó un total de 300 encuestas. De estas se desprende que un 99,3\% $(94,6 \%)$ de la población no acepta vivir en las cercanías (a menos 600 metros) de un RS (ET). Pero el 99,6\% y el $95,0 \%$, para cada uno de los equipamientos mencionados, reconoce que éstos son necesarios. Con estas respuestas es posible afirmar que los Rellenos y las Estaciones de Transferencia cumplen con la lógica de una Actividad Socialmente Indeseable.

Otro resultado de la encuesta fue el modelo de porcentaje de devaluación en función de la distancia. Para el caso de Rellenos Sanitario se calibró un modelo lineal con la variable explicativa de la distancia. Pero, para el caso de Estaciones de Transferencia y debido a la escasez de datos encontrados, se estimó sólo con un ajuste de curva.

Los estadígrafos muestran un buen comportamiento del modelo econométrico, tanto en significancia como en ajuste (R2 adj=0,6).

De los modelos se puede deducir una relación inversa entre la distancia y el devalúo, es decir, a mayor distancia menores son los efectos percibidos. En un radio de 14,9 $\mathrm{km}$ la externalidad tiende a ser cero. El máximo devalúo estimado por el modelo a distancia cero es de 78,3\%. En el modelo de ET también se presenta una relación inversa entre la distancia y el devalúo, resultando para este caso un radio de influencia de $1,4 \mathrm{~km}$, y un porcentaje de devaluación máximo (estimado) de 74,8\%, similar al de RS. 
Para la estimación de RSD mensual a nivel comunal se calibró un modelo del tipo ARIMA para cada comuna y para la región en su totalidad.

Así se obtuvo una proyección para la Región Metropolitana de un total de 49.374.363 toneladas durante los próximos 15 años, lo que significa que en promedio serán 3.291.624 toneladas anuales.

Los valores de Ponderadores determinados con el método Delphi corresponden al nivel de importancia considerando una perspectiva técnica, ya que el Panel de Expertos está compuesto por especialistas técnicos en el Área de Planificación Territorial, Residuos Sólidos Domiciliarios y Medio Ambiente. Así se determinó una solución que privilegia los criterios económicos $(\mathrm{P} 1=0,57)$ sobre los sociales $(\mathrm{P} 2=0,43)$.

Con todo lo anterior, y en base a la estrategia de modelación establecida, se aplicó el modelo de optimización. La solución del modelo entrega dos tipos de resultados. El primero corresponde a la selección de Sitios para localizar

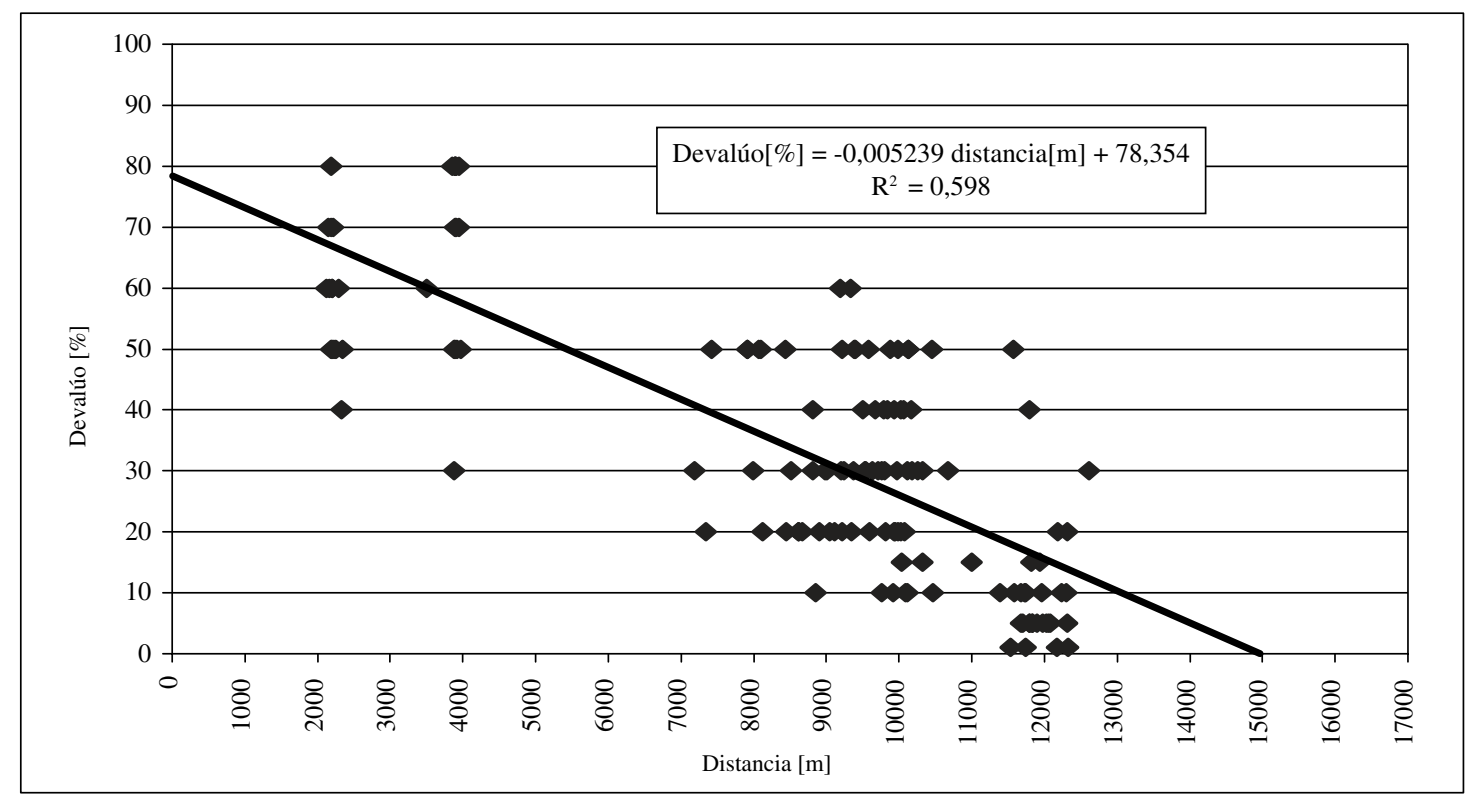

Figura 4. Regresión Lineal: Devalúo v/s Distancia para Rellenos Sanitarios.

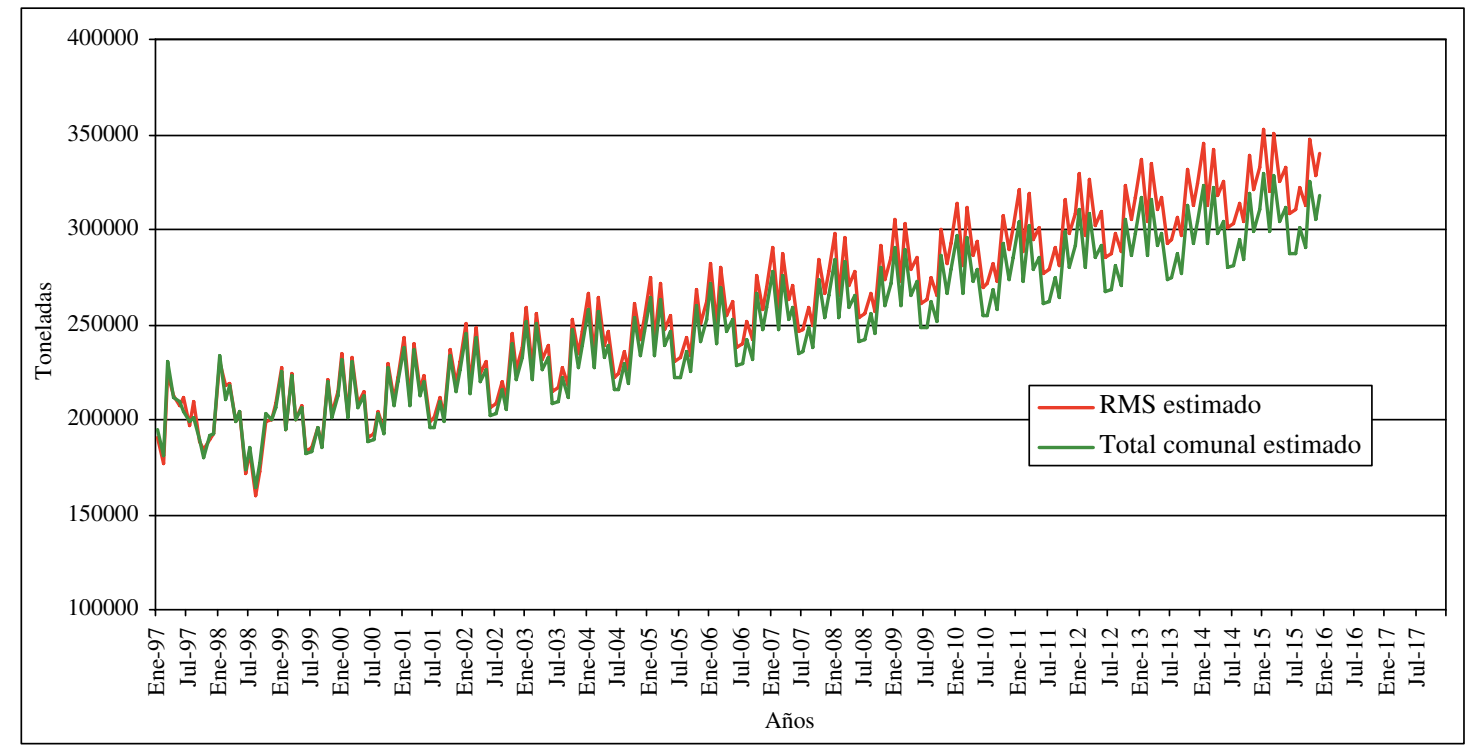

Figura 5. RSD mensuales para la RM. 
Tabla 1. Elección de sitios para RS y ET de la solución óptima.

\begin{tabular}{|c|c|c|c|c|c|c|}
\hline \multirow[b]{2}{*}{ Id } & \multirow[b]{2}{*}{ Equipamiento } & \multirow[b]{2}{*}{ Comuna } & \multirow[b]{2}{*}{ Código } & \multirow[b]{2}{*}{$\begin{array}{c}\text { Alternativa } \\
\text { Tamaño }\end{array}$} & \multirow{2}{*}{ 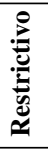 } & \multirow{2}{*}{ בְב } \\
\hline & & & & & & \\
\hline $\mathrm{A}$ & ET Z.I.E. Puente Alto & Puente Alto & $\mathrm{Z} 11$ & 1 & & \\
\hline $\mathrm{A}$ & ET Z.I.E. Puente Alto & Puente Alto & $\mathrm{Z} 12$ & 2 & & \\
\hline A & ET Z.I.E. Puente Alto & Puente Alto & Z13 & 3 & & \\
\hline B & ET Z.I.E. Lo Espejo & San Bernardo & $\mathrm{Z} 21$ & 1 & $\bullet$ & \\
\hline B & ET Z.I.E. Lo Espejo & San Bernardo & $\mathrm{Z} 22$ & 2 & & \\
\hline $\mathrm{B}$ & ET Z.I.E. Lo Espejo & San Bernardo & $\mathrm{Z} 23$ & 3 & & \\
\hline $\mathrm{C}$ & ET Ex Pozo Lo Errázuriz & Cerrillos & $\mathrm{Z31}$ & 1 & & \\
\hline $\mathrm{C}$ & ET Ex Pozo Lo Errázuriz & Cerrillos & $\mathrm{Z} 32$ & 2 & & \\
\hline $\mathrm{C}$ & ET Ex Pozo Lo Errázuriz & Cerrillos & $\mathrm{Z33}$ & 3 & & \\
\hline $\mathrm{E}$ & ET Z. Extracc. Áridos & Puente Alto & Z41 & 1 & & \\
\hline $\mathrm{E}$ & ET Z. Extracc. Áridos & Puente Alto & $\mathrm{Z} 42$ & 2 & & \\
\hline$E$ & ET Z. Extracc. Áridos & Puente Alto & Z43 & 3 & $\bullet$ & $\bullet$ \\
\hline $\mathrm{J}$ & ET Z.I.E. Quilicura & Quilicura & $\mathrm{Z} 51$ & 1 & & \\
\hline $\mathrm{J}$ & ET Z.I.E. Quilicura & Quilicura & $\mathrm{Z} 52$ & 2 & & \\
\hline $\mathrm{J}$ & ET Z.I.E. Quilicura & Quilicura & Z53 & 3 & $\bullet$ & $\bullet$ \\
\hline $\mathrm{Q}$ & ET Quilicura & Quillcura & Z64 & 4 & $\bullet$ & $\bullet$ \\
\hline PS & ET Puerta Sur & San Bernardo & $\mathrm{Z75}$ & 5 & $\bullet$ & $\bullet$ \\
\hline 8 & RS Cuesta Chada & Paine & Y11 & 1 & & \\
\hline 8 & RS Cuesta Chada & Paine & Y12 & 2 & & \\
\hline 8 & RS Cuesta Chada & Paine & Y13 & 3 & $\bullet$ & $\bullet$ \\
\hline 19 & RS Sector Paso de la Arena & Melipilla & Y21 & 1 & & \\
\hline 10 & RS Sector Paso de la Arena & Melipilla & Y23 & 3 & & \\
\hline 20 & RS San Diego de Melipilla & Melipilla & Y31 & 1 & $\bullet$ & $\bullet$ \\
\hline 20 & RS San Diego de Melipilla & Melipilla & Y33 & 3 & & \\
\hline 30 & RS Sector Sur Cerro Camero & Maipú & Y41 & 1 & & \\
\hline 30 & RS Sector Sur Cerro Camero & Maipú & Y42 & 2 & & \\
\hline 30 & RS Sector Sur Cerro Camero & Maipú & Y43 & 3 & & \\
\hline 31 & RS Cerro Camero & Pudahuel & Y51 & 1 & & \\
\hline 31 & RS Cerro Camero & Pudahuel & Y52 & 2 & & \\
\hline 31 & RS Cerro Camero & Pudahuel & Y53 & 3 & & \\
\hline 37 & RS C.R.I.O.T. Peldehue & Colina & Y61 & 1 & & \\
\hline 37 & RS C.R.I.O.T. Peldehue & Colina & Y62 & 2 & & \\
\hline 37 & RS C.R.I.O.T. Peldehue & Colina & Y63 & 3 & & \\
\hline 38 & RS Peldehue & Colina & Y71 & 1 & & \\
\hline 38 & RS Peldehue & Colina & Y72 & 2 & & \\
\hline 38 & RS Peldehue & Colina & Y73 & 3 & & \\
\hline LC & RS Loma Los Colorados & Til Til & Y84 & 4 & $\bullet$ & $\bullet$ \\
\hline SM & RS Santa Marta & Talagante & Y95 & 5 & $\bullet$ & $\bullet$ \\
\hline SP & RS Santiago Poniente & Maipú & Y106 & 6 & $\bullet$ & $\bullet$ \\
\hline
\end{tabular}

RS y ET, y el segundo corresponde a la distribución y asignación de residuos desde las Zonas Generadoras (ZG) a las ET y/o RS, como también, desde las ET a los RS.

En la tabla 1 se muestran los sitios elegidos por el modelo en los dos escenarios para el período 15 años. La selección de sitios y alternativas de tamaños es similar entre el Escenario Libre y Restrictivo, con la excepción del Sitio B para localizar una Estación de Transferencia en la comuna de Lo Espejo. Esto demuestra la rigidez del Escenario Restrictivo, ya que los residuos comunales son destinados directamente al Relleno Sanitario, o bien, a una Estación de Transferencia, justificándose este escenario cuando la solución encontrada distribuye sus residuos en partes mínimas haciendo poco operativo al Sistema.

El hecho que el Escenario Libre seleccione un equipamiento menos que el Escenario Restrictivo produce un Valor de la Función Objetivo menor y, por ende, un menor Costo para el Sistema, por lo tanto, la selección de Sitios para instalar Rellenos Sanitarios y Estaciones de Transferencia óptima que será propuesta, corresponderá a la configuración dada por el Escenario Libre. Ver tabla 2. 
Tabla 2. Valor de la Función Objetivo para cada escenario, en periodo de Estudio (15 años).

\begin{tabular}{|l|l|l|}
\hline & Ponderadores & Función Objetivo \\
\hline $\begin{array}{l}\text { Escenario } \\
\text { Restrictivo }\end{array}$ & $\mathrm{P} 1=0,571$ & $\mathrm{MM} \$ 1.347 .060,0$ \\
\cline { 1 - 1 } $\begin{array}{l}\text { Escenario } \\
\text { Libre }\end{array}$ & $\mathrm{P} 2=0,429$ & $\mathrm{MM} \$ 1.248 .256,4$ \\
\hline
\end{tabular}

Las asignaciones óptimas se muestran en la siguiente figura, en donde coexisten comunas que se asignan sólo a RS, sólo a ET y mixtas, es decir, parte a RS y parte a ET (Quinta Normal, Cerro Navia y El Bosque).

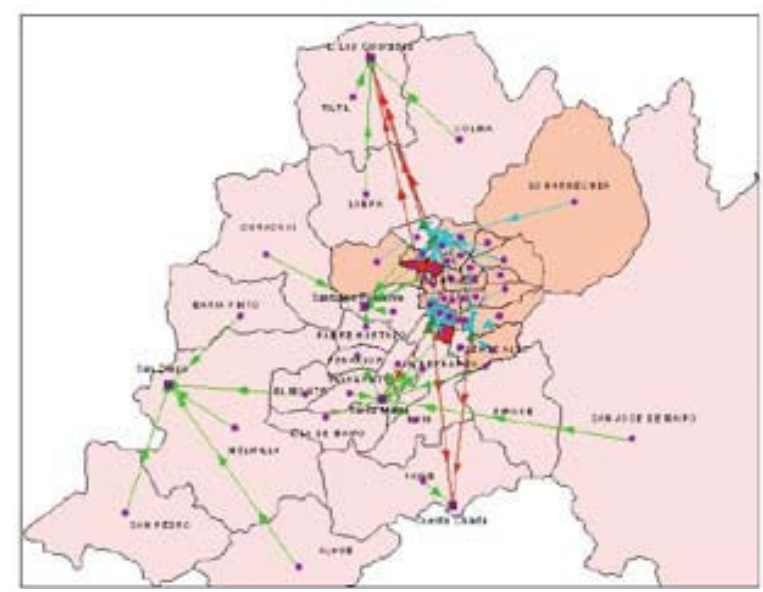

Figura 6. Asignación óptima de RSD a RS y ET.

Respecto al análisis de sensibilidad de los parámetros P1 y P2 se aplicó el modelo para el caso absolutamente económico $(\mathrm{P} 1=1$ y $\mathrm{P} 2=0)$, caso absolutamente social $(\mathrm{P} 1=0$ y $\mathrm{P} 2=1)$ y en situaciones intermedias $(\mathrm{P} 1=0,25$ y $\mathrm{P} 2=0,75 ; \mathrm{P} 1=0,5$ y $\mathrm{P} 2=0,5$ y $\mathrm{P} 1=0,75$ y $\mathrm{P} 2=0,25)$.

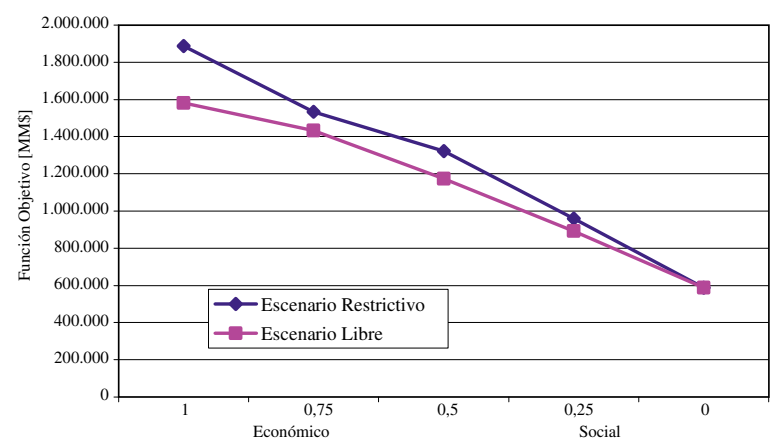

Figura 7. Variación de la F.O., al sensibilizar parámetros P1 y P2.

En la figura 7 se aprecia cómo varía el valor de la F.O. cuando se pasa de la situación sólo económica (izquierda) a la situación sólo social (derecha), para el período de 15 años. Cabe destacar que siempre el escenario libre es mejor respecto que el restrictivo, y que el óptimo sólo social es menor (50\%) que el solo económico. Respecto de los sitios elegidos, existe variabilidad sólo en un candidato del escenario sólo económico respecto del sólo social, lo que indica la relativa robustez del modelo, respecto de la localización espacial.

\section{CONCLUSIONES}

- La externalidad social, referida al devalúo de las viviendas, presenta una relación inversa con la distancia, afectando, para el caso de un RS hasta un radio de $14,9 \mathrm{~km}$, y $1,4 \mathrm{~km}$ para una ET. Los máximos devalúos son $78,3 \%$ a distancia cero de un RS, y $74,8 \%$ a distancia cero de una ET.

- El costo mínimo para el Sistema, durante el periodo de estudio 2004-2018, es de MM\$ 1.248.256,4. Este valor corresponde a la configuración de sitios del Escenario Libre, que fue el seleccionado, ya que presenta un costo que es un $7 \%$ menor que el Escenario Restrictivo.

- La configuración de sitios seleccionados del Escenario Libre contempla la incorporación de dos nuevos sitios para instalar Rellenos Sanitarios: el sitio San Diego en Melipilla y el sitio Cuesta Chada en Paine.

- Considerando todo lo señalado, el Modelo de localización-asignación propuesto, implementado como un SIG es una herramienta muy útil para el Planificador Territorial, ya que permite obtener resultados considerando diversas variables que van desde lo económico a lo social, además de establecer diversos escenarios que permiten una modelación para problemas específicos.

- Finalmente, y según los datos obtenidos, un Relleno Sanitario o una Estación de Transferencia son considerados como Actividades Socialmente Indeseables, siendo habitualmente rechazada la instalación de estos equipamientos por parte de la población (Síndrome NIMBY). Debido a esto, el factor Social juega un papel muy importante dentro de la localización de estas Actividades. Por este hecho, la incorporación al Modelo propuesto, del Principio de Justicia Espacial en conjunto con el Principio de Eficiencia, permite encontrar una configuración espacial de equipamientos que genere el menor costo posible, tanto Económico como Social, optimizando el Sistema de Transporte y Disposición Final y, por ende, el Sistema en su globalidad. 


\section{REFERENCIAS}

[1] J. Bosque. "Sistemas de Información Geográfica". Rialp Editores. Edición 2. Madrid, España. Vol. 1. 1997.

[2] J. Bosque, M. Gómez, A. Moreno y F. dal Pozzo. "Hacia un Sistema de Ayuda a la Decisión Espacial para la Localización de Equipamientos". Publicado en Estudios geográficos 2000. Tomo LXI No 241, pp. 567-598. 2000.

[3] J. Bosque y A. Moreno. "Sistema de Información Geográfico y localización óptima de instalaciones y equipamientos". Editorial RA-MA. Edición 1. Madrid, España. 2004.

[4] CIS Consultores. "Análisis Vial de Sitios Potenciales, Localización de R.S.D”. Estudio técnico encargado por el Gobierno Regional Metropolitano. Santiago, Chile. 2001.

[5] S. De la Torre. "Análisis del Mercado de Recolección de Residuos Sólidos Domiciliarios, Provincia de Santiago, 1990-1998”. Departamento de Ingeniería Industrial. Tesis para optar al grado de Ingeniero Civil Industrial. Universidad de Chile. Santiago, Chile. 2000.

[6] E. Erkut, S. Neuman. "Analytical models for locating undesirable facilities". European Journal of Operational Research. Vol. 40 No 3, pp. 275-291. Junio 1989.

[7] E. Erkut, S. Neuman. "A multiobjective model for locating undesirable Facilities". Annals of Operations Research. Volume $40 \mathrm{~N}^{\mathrm{os}} 1-4$, pp. 209227. Diciembre 1992.

[8] Gobierno Regional de la Región Metropolitana. "Propuesta de Localización de Zonas para la
Instalación de Rellenos Sanitarios y Estaciones de Transferencia en la Región Metropolitana". Estudio técnico encargado por el Gobierno Regional Metropolitano. Santiago, Chile. 1999.

[9] S. Gómez. "Funciones de Costos y Consideraciones Técnicas para Sistemas de Manejo de Residuos Sólidos en Comunidades Pequeñas”. Tesis para optar al grado de Ingeniero de Ejecución en Ambiente. Universidad de Santiago de Chile. Santiago, Chile. 2000.

[10] C. Guerrero, G. Silva. "Diagnóstico de los sitios de Disposición Final de los Residuos Sólidos Domiciliarios, Diseño e Implementación de un Sistema de Calificación Ambiental de estos sitios en la Quinta Región”. Tesis para optar al grado de Ingeniero de Ejecución en Ambiente. Universidad de Santiago de Chile. Santiago, Chile. 2000.

[11] A. Moreno. "Una familia de Modelos de Localización Óptima para Actividades Indeseables: propuesta y aplicación con S.I.G”. Working Paper, Depto. de Geografía, Universidad Autónoma de Madrid. 1998.

[12] C. Pérez. "Técnicas Estadísticas con SPSS". Editorial Prentice Hall. Madrid, España. 571 páginas. 2004.

[13] G. Solís. "Estudio de prefactibilidad técnica económica y ambiental de una Estación de Transferencia de Residuos Sólidos Domiciliarios para la Zona Sur de la Región Metropolitana de Santiago". Tesis para optar al grado de Ingeniero Civil Mecánico. Universidad de Chile. Santiago, Chile. 2001.

[14] G. Tchobanoglous, H. Theisen, S. Vigil. "Gestión Integral de Residuos Sólidos”. Editorial McGrawHill. Vols. 1 y 2. Madrid, España. 1994. 